\title{
Combined HST/STIS, FUSE, and Chandra observations of the Seyfert 1 galaxy NGC 4151
}

\author{
S. B. Kraemer ${ }^{1}$, D. M. Crenshaw ${ }^{2}$, \\ J. R. Gabel ${ }^{3}$ and I. M. George ${ }^{4}$ \\ ${ }^{1}$ Department of Physics, Catholic University of America, Washington, DC 20064, USA \\ email:kraemer@yancey.gsfc.nasa.gov \\ ${ }^{2}$ Department of Physics and Astronomy, Georgia State University, Atlanta, GA 30303 \\ ${ }^{3}$ University of Colorado, CASA, Boulder, CO 80309 \\ ${ }^{4}$ JCA, University of Maryland, Baltimore County, Baltimore, MD 21250
}

\begin{abstract}
We report the results of combined Chandra, HST/STIS, and FUSE observations of the intrinsic absorption in the Seyfert 1 galaxy NGC 4151. Our previous studies revealed an unusually large column of outflowing gas, very close to the nucleus, which responds rapidly to changes in the ionizing continuum due to its high density. With the latest observations, we have discovered evidence that the large absorbing column consists of individual high and low ionization components, and that the column density of the latter has decreased due to bulk motion across our line-of-sight. The Chandra spectra have revealed the signature of both very high ionization lines such as $\mathrm{H}$-like and $\mathrm{He}$-like $\mathrm{Si}$, and inner-shell lines from low ionization species which may be associated with the UV absorbers. With the FUSE observations, we have detected lines from less abundant elements, which have permitted us to constrain the physical conditions and covering factors of the UV absorbers. These results indicate a complex and heterogeneous absorbing system, the extreme properties of which are likely the result of a relatively large viewing angle with respect to the accretion-disk axis.
\end{abstract}

\section{Introduction}

Blueshifted absorption lines in the UV and X-ray spectra of active galaxies reveal the presence of massive outflows of ionized gas from their nuclei (Crenshaw, Kraemer, \& George 2003, and references therein). UV observations of the Seyfert 1 galaxy NGC 4151, initially by the IUE (Boksenberg et al. 1978) revealed a number of absorption lines, including those from neutral and low ionization states, e.g. O I, Fe II, and Mg II. Early X-ray observations showed the presence of a large column of atomic gas (e.g. Barr et al. 1977), which is now known to be ionized (e.g. George et al. 1998). Both the $\mathrm{UV}$ and X-ray absorption are variable, due to changes in the ionizing continuum (e.g. Bromage et al. 1985) and, possibly, bulk motion across the line-of-sight (George et al. 1998). Observations with the HST/FOS and GHRS found UV kinematic components at velocities $-1580,-840,-500,-250,-200,0$, and $+50 \mathrm{~km} \mathrm{~s}^{-1}$ with respect to systemic (dubbed components A, C, D, E, E', F, and $\mathrm{F}^{\prime}$ by Weymann et al. 1997). We observed NGC 4151 with HST/STIS, using the E140M and E230M echelles, on 1999 July 19, during which time the continuum flux was in a low state $(\sim 25 \%$ peak). Large column densities from low ionization species were found associated with the absorption complex of components D+E, including lines from metastable Fe II and C III (Kraemer et al. 2001). We argued that the presence of strong low ionization absorption was a consequence of 


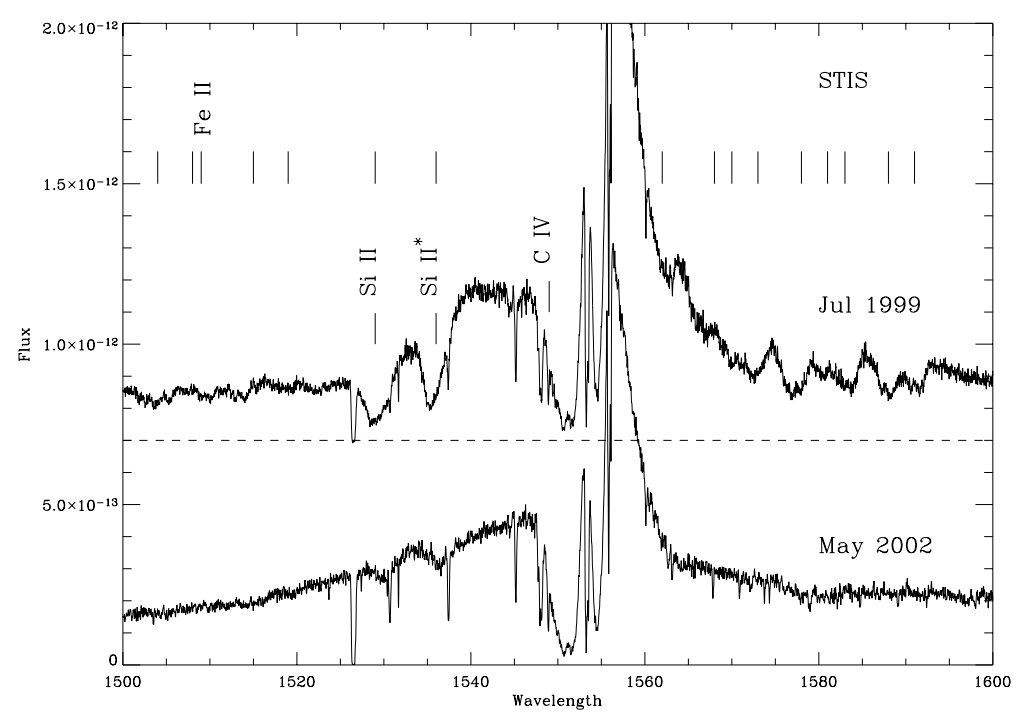

Figure 1. A comparison of the 1999 July and 2002 May STIS/E140M spectra of NGC 4151 in the region near C IV $\lambda 1550$. The vertical tick marks show the location of absorption lines from metastable Fe II states. Note the absence of the Fe II absorption and comparative weakness of the Si II and Si II* lines in the later spectrum.

the weak ionizing flux. We were able to derive ionization parameters ( $\mathrm{U}$, the number of ionizing photons per $\mathrm{H}$ atom) for the UV components. Using the density constraints from the metastable lines, we determined that the $\mathrm{D}+\mathrm{E}$ gas was composed of a large column of gas $\left(\mathrm{N}_{H} \sim\right.$ few x $\left.10^{21} \mathrm{~cm}^{-2}\right)$ within $0.03 \mathrm{pc}$ of the central source. However, the lack of simultaneous X-ray observations prevented us from probing the connection of the X-ray and UV absorbers. To pursue this, we reobserved NGC 4151 with the HST/STIS and FUSE on 2002 May 8 and the Chandra/High Energy Transmission Grating (HETG) on 2002 May 9.

\section{New Results and Conclusions}

Our 2002 May observations of NGC 4151 found the UV continuum flux nearly identical to that of 1999 July. Hence, we expected to find little change in the intervening absorption. However, as shown in figure 1, the strong low-ionization lines, including the myriad of lines from metastable Fe II, were weak or absent in the later spectra. The most straightforward explanation is that a large fraction $(\sim 50 \%)$ of the gas associated with $\mathrm{D}+\mathrm{E}$ must have moved out of our line of sight. Note that there is strong evidence for transverse motion in the absorbers in other Seyfert 1s (Kraemer, Crenshaw, \& Gabel 2001, Crenshaw et al. 2003). While the low-ionization absorption decreased, higher ionization UV lines such as C IV $\lambda 1548,1551, \mathrm{~N}$ V $\lambda \lambda 1239,1243$, and O IV $\lambda \lambda 1032,1037$ remained saturated at these velocities, with covering fractions of the combined continuum and broad-line emission $\gtrsim$ 0.8. Although we expected these lines to be saturated, we had hoped to better constrain the physical conditions in component $\mathrm{D}+\mathrm{E}$ using lines from less abundant elements that co-exist with the CNO Li-like ions, specifically P V $\lambda \lambda 1118,1128$. While the $\mathrm{P}$ V lines were unsaturated, we derived a cover factor of $\sim 0.5$ from the doublet, which suggests that there are at least two components contributing to the absorption at this velocity.

The Chandra/HETG spectra revealed that the continuum flux increased by a factor of $\sim 2.5$ compared to the Chandra GTO spectrum obtained in 2000 March 5 (Ogle et al. 


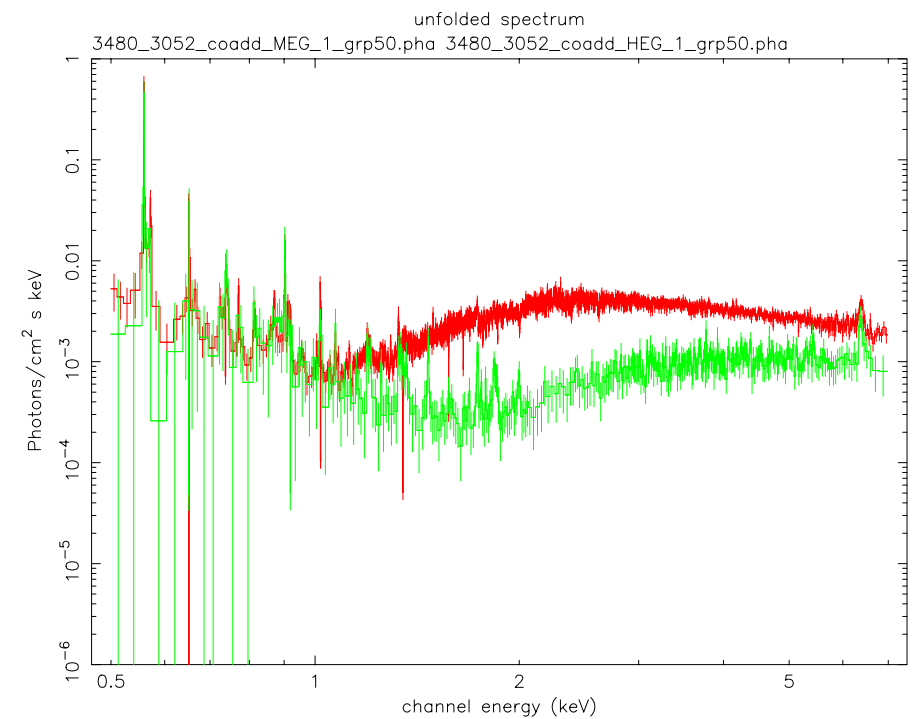

Figure 2. A comparison of our HETG spectrum from 2002 May (red) and the GTO spectrum from 2000 March (green). Note that emission lines dominate the spectra below $\sim 1 \mathrm{keV}$, and that the soft emission is essentially identical in the two epochs. The hard X-ray flux was $\sim 2.5$ times stronger in 2002, primarily due an increase in luminosity of the central source.

2000), as shown in figure 2. Interestingly, in both observations the flux below $\sim 1 \mathrm{keV}$ is dominated by emission lines, and the soft X-ray flux has not changed significantly over this time period. This suggests that 1 ) the soft X-ray lines are formed far enough away (e.g. $\gtrsim 1 \mathrm{pc}$ from the central source) that they have not responded to the increase in the continuum flux and 2) the photo-electric edge is deeper than it appears in the 2002 spectrum, since it is partially filled-in by the extended soft emission. Numerous absorption lines from $\mathrm{Mg}$, Si and $\mathrm{S}$ were present in the HEG and MEG spectra (figure 3). Innershell lines from the L-shell states of these ions are most likely formed in the same gas that produces the strong Li-like CNO lines, and, indeed, the X-ray absorption spans the same range in velocity as the UV absorbers. Most interesting is the presence of strong $\mathrm{H}$-like lines from $\mathrm{S}$ and $\mathrm{Si}$. The fact that these are clearly stronger than the He-like lines (see figure 3) indicates the presence of a very highly ionized component, which may be the source of the scattered light in the troughs of the saturated UV lines (Kraemer et al. 2001). Furthermore, the H-like lines span the same range of velocities as the lower ionization lines.

Based on our preliminary photoionization models, we find that the the $\mathrm{D}+\mathrm{E}$ complex consists of at least 3 individual components. The $\mathrm{P} \mathrm{V}$ lines arise in a low-covering factor component characterized by $\mathrm{U} \sim 0.1$ and $\mathrm{N}_{H} \sim 5 \mathrm{x} 10^{21} \mathrm{~cm}^{-2}$. The high covering factor absorption arises in two components characterized as follows: $\mathrm{U} \sim 0.01, \mathrm{~N}_{H} \sim$ few $\mathrm{x}$ $10^{20} \mathrm{~cm}^{-2} ; \mathrm{U} \sim 0.5, \mathrm{~N}_{H} \sim$ few $\mathrm{x} 10^{22} \mathrm{~cm}^{-2}$. The metastable Fe II lines most likely were formed in the former, when its column density was greater. The higher ionization component produces the saturated CNO lines, and is the source of the inner shell $\mathrm{S}$ and Si lines and the X-ray photo-electric cutoff. In addition, another component of very highly ionized gas $(\mathrm{U} \gtrsim 10)$ is required to produce the $\mathrm{H}$ - and He-like $\mathrm{S}$ and Si lines. This last component, although it is clearly blue-shifted, is too highly ionized to be driven by radiation pressure, although could be part of a hydromagnetic flow (e.g., Bottorff, Korista, \& Shlosman 2000) or thermal wind (e.g., Begelman, McKee, \& Shields 1983). 


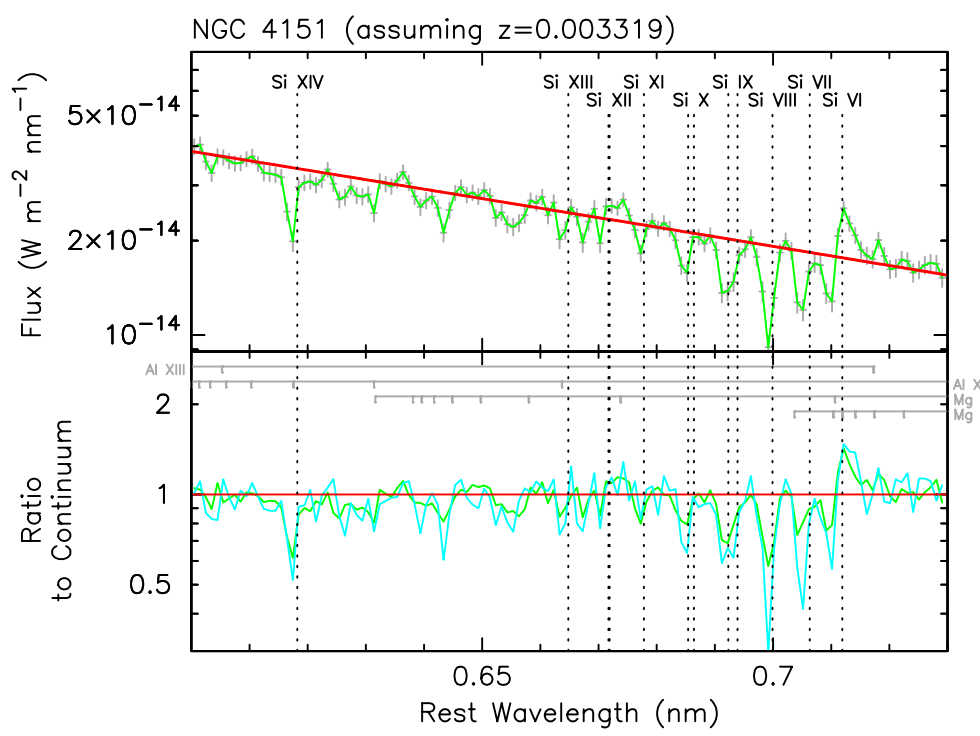

Figure 3. A portion of the combined MEG and HEG spectra of NGC 4151, showing absorption lines from Si XIV (H-like), Si XIII (He-like), and inner shell lines from lower ionization states of silicon. The red-line is included to help show the absorption lines, but is not intended to represent the intrinsic continuum shape. The vertical lines are the rest wavelengths of the $\mathrm{Si}$ lines (with respect to the systemic velocity of NGC 4151), showing that the lines are blue-shifted (indicative of outflow).

Determining how these components are related is crucial to understanding the origin of such outflows. The complexity of the absorption is likely a result of the fact that we are viewing the putative accretion disk in NGC 4151 at high inclination, which suggests that the characteristics of the flow depend strongly on viewing angle.

\section{Acknowledgements}

S.B.K., J.R.G, and I.M.G. acknowledge support from Smithsonian GO2-3138A.

\section{References}

Barr, P., et al. 1977, MNRAS, 181, 43P

Begelman, M. C., McKee, C. F., \& Shields, G. A. 1983, ApJ, 271, 70

Boksenberg, A., et al. 1978, Nature, 275, 404

Bottorff, M. C., Korista, K. T., \& Shlosman, I. 2000, ApJ, 537, 134

Bromage, G. E., et al. 1985, MNRAS, 251, 1

Crenshaw, D. M., Kraemer, S. B., \& George, I. M. 2003, ARA\&A, 41, 117

Crenshaw, D. M., et al. 2003, ApJ, 594, 116

George, I. M., et al. 1998, ApJS, 114, 73

Kraemer, S. B., et al. 2001, ApJ, 551, 671

Kraemer, S. B., Crenshaw, D. M., \& Gabel, J. R. 2001, ApJ, 557, 30

Ogle, P. M., et al. 2000 ApJ, 545, L81

Weymann, R. J., et al. 1997, ApJ, 483, 717 